\title{
Comunicación estratégica: modelo de gestión comunicacional
}

\author{
Patricia Durán Bravo ${ }^{1 *}$, Nancy G. Cisneros Martínez², Víctor M. Meléndez Rodríguez ${ }^{3}$, Rafael \\ Cid Quiroz ${ }^{4}$
}

\begin{abstract}
Resumen
Las transformaciones sociales, tecnológicas y globales han provocado un reto complejo para las organizaciones; por una parte, la competitividad que exige el cumplimiento de sus objetivos institucionales; y por otra, la sustentabilidad que demanda la trascendencia y el valor social de sus acciones. Para mejorar los resultados de la gestión de la comunicación en la creación de valor organizacional, se presenta el Modelo ICA, que integra diversas técnicas de diagnóstico, implementación y evaluación, que permitirá gestionar el sistema comunicacional de la organización desde una perspectiva sistémica, estratégica y socio-comunicacional. Gestionar la comunicación con el Modelo ICA permitirá un diagnóstico estructural, funcional, relacional y simbólico de la comunicación de la organización para el desarrollo de una Estrategia Comunicacional Corporativa (ECCo) que aporte valor estratégico a la organización.

\section{Abstract}

Social, technological and global transformations have created a complex challenge for organizations; on the one hand, the competitiveness that demands the fulfillment of its institutional objectives; and on the other, the sustainability that demands the transcendence and social value of its actions. To improve the results of communication management in the creation of organizational value, the ICA Model is presented, which integrates various diagnostic, implementation and evaluation techniques, which will allow the organization's communication system to be managed from a systemic, strategic and strategic perspective. socio-communicational Managing communication with the ICA Model will allow a structural, functional, relational and symbolic diagnosis of the organization's communication for the development of a Corporate Communication Strategy (ECCo) that provides strategic value to the organization.

\section{Palabras Clave}

Comunicación Estratégica, Gestión comunicacional, Organizaciones.

Keywords

Strategic Communication, Communication Management, Organizations.

${ }^{1}$ Doctora en Comunicación. Docente de la Maestría en Comunicación Estratégica, líder del cuerpo académico de comunicación en las organizaciones, de la Facultad de Comunicación de la BUAP.

${ }^{2}$ Doctora en Imagen y Estética. Docente de la Maestría en Comunicación estratégica, Miembro del Cuerpo Académico Comunicación en la Organizaciones de la Facultad de Ciencias de la Comunicación de la BUAP.

${ }^{3}$ Maestro en Administración. Docente de la Maestría en Comunicación Estratégica, miembro del cuerpo académico de comunicación en las organizaciones de la Facultad de Comunicación de la BUAP.

${ }^{4}$ Maestrante del posgrado en Comunicación Estratégica, colaborador del cuerpo académico de comunicación en las organizaciones de la Facultad de Comunicación de la BUAP.

*Autor para correspondencia: patricia.duran@correo.buap.mx
\end{abstract}

\section{Introducción}

El entorno social de las organizaciones es un mapa relacional que se conforma por múltiples y diversos vínculos 
integrados en una red global, en el que se articulan dinámicas sociales convergentes y divergentes, que interactúan y se influyen de manera continua, no sólo a nivel funcional sino también a nivel simbólico, esta circunstancia somete a la organización a una permanente tensión rodeada de una complejidad que, además, la enfrenta a diferentes problemáticas en el ámbito comunicacional. Estas se originan debido a ciertas características del entorno actual, entre las que se pueden identificar:

1. Un ambiente de convergencia de discursos en los que se expresan: demandas, necesidades, exigencias y expectativas de sus grupos de interés (empleados, proveedores, clientes, autoridades, sociedad, entre otros).

2. Un complejo metabolismo social cargado de hiperconectividad digital, que agiliza el intercambio informativo, la producción de mensajes y la interacción global.

3. Multiplicidad de vínculos con y entre los grupos de interés, no sólo funcionales (ventas, transacciones financieras, suministros, etc.), sino también vínculos afectivos y emocionales, que provocan una necesidad a debatir, exigir, conversar, seducir y convencer a todos sus públicos estratégicos.

4. Procesos de significación sofisticados que buscan la apropiación simbólica y materialización de los recursos tecnológicos (productos y servicios), sociales (atención al cliente) y culturales (responsabilidad social) de la organización.

Actualmente, las organizaciones buscan comunicarse con sus grupos de interés a los que no les basta con recibir información; su demanda gira en torno a un esfuerzo de gestión comunicacional que forme vínculos de alta densidad para lograr su competitividad sustentable. Para ello, es indispensable la gestión estratégica de su sistema comunicacional que permita la generación de valor, a través de la articulación de la producción simbólica de sus grupos de interés a los propósitos organizacionales.

En este sentido, "los paradigmas tradicionales de gestión organizacional centrados en el reduccionismo, la simplicidad y el estatismo, hoy no responden a las demandas del entorno" (Pérez. 2012 p. 137). Es necesario recordar que los sistemas organizacionales son entidades complejas que requieren algo más que sólo el manejo de sus recursos materiales, financieros y humanos; para lograr la generación de valor estratégico en las organizaciones, es indispensable integrarindispensable integrar, además, los recursos sociales, culturales y simbólicos. Se propone un modelo de gestión comunicacional que logre solucionar de manera estratégica los problemas simbólicos, relacionales y de articulación, que permita a la organización obtener resultados sustentables.

\section{Potencialización de la producción simbólica: los activos intangibles organizacionales}

La comunicación estratégica es la articulación de la producción simbólica del sistema social a los propósitos organizacionales, para generar valor a nivel individual, organizacional y social. Desde esta perspectiva, las organizaciones son realidades en construcción, que para lograr visiones colectivas generan posibilidades de actuación deliberadas, sistemáticas y relacionantes entre los múltiples componentes del sistema organizacional. Nosnik (2012) denomina "sistemas de convivencia a los grupos humanos que se organizan para cumplir con un propósito o fin común; cuando éstos son formales se les llama organizaciones" ( $\mathrm{p}$. 13).

Las organizaciones son esencialmente un sistema de comunicación, en el que interactúan sus integrantes para desarrollar procesos de consenso, mediación y negociación (Rodríguez y Opazo, 2007). Para lograr su eficiencia se propone un sistema de gestión comunicacional sistémico y estratégico, cuyo centro es la capacidad de aprendizaje, creatividad e innovación, que surgen en la diversidad de interacciones de la organización con sus grupos de interés. Para Pérez (2012) la comunicación estratégica en una organización logra su efectividad en la medida que se incorporan los procesos de significación, las relaciones humanas y la proyección de futuro en sus actividades funcionales.

Para gestionar la realidad organizacional desde la comunicación estratégica, es importante reconocer una 
dualidad: el papel que desempeña la estructura y/o diseño institucional, así como, el rol activo del agente organizacional. Es decir, que para gestionar la comunicación estratégica se debe analizar de manera dialógica la forma en que estos agentes realizan intercambios con su estructura, a través de sus representaciones simbólicas y acciones operativas, construidas socialmente en condiciones específicas (Bourdieu, 2002).

Para Bourdieu y Wacquant (1995), la construcción de las representaciones sociales depende de la cantidad y tipo de información que intercambian, el contexto sociocultural en el que se desarrolla ese intercambio y la posición social de los agentes dentro de la estructura organizacional. Estos sistemas se convierten en una "matriz simbólica de las actividades, prácticas, conductas, pensamientos, sentimientos y juicios de los agentes sociales" (pp. 18).

Parsons (2012) afirma que para lograr la sustentabilidad del sistema organizacional deben orientarse dos operaciones básicas:

1. La integración: capacidad de compartir los mismos valores en su estructura.

2. La adaptación: capacidad de resolver diferenciadamente sus problemas.

La gestión del sistema de comunicación en una organización genera valor compartido, en la medida en la que hace complementarias estas dos funciones: integración y adaptación. En primera instancia, la integración holística debe considerar los subsistemas que Parsons (2012) define como: "el cultural: necesidades orgánicas y emocionales; el social: interacción o interdependencia de los agentes; y el de personalidad: patrones simbólicos, de sentido o valor" (p. 94). En segunda, el sistema de comunicación debe articular la producción simbólica de una gran diversidad de grupos, agentes y otros sistemas, a los propósitos organizacionales, de tal manera que se genere una red de valor con impacto a nivel micro, supra y macro-sistema.

Se requieren métodos y herramientas que sistematicen la función comunicacional de todos y cada uno de los componentes del sistema organizacional y que, además, permitan articular los activos tangibles e intangibles así como los propósitos organizacionales. De tal manera que se produzca valor al contenido de los intercambios: orientando las representaciones sociales, moldeando el comportamiento organizacional y creando "ambientes ricos en información" (Nosnik, 2012).

La producción simbólica se expresa en los activos intangibles organizacionales que Kaplan y Norton (2004) categorizan en tres niveles:

1. Capital humano: destrezas, talento, aprendizaje, sentido de pertenencia, motivación, creatividad e innovación, etc.

2. Capital de información: bases de datos, sistemas de información, redes y flujos informativos, etc.

3. Capital organizacional: cultura, clima, liderazgo, trabajo en equipo, opinión pública, imagen y reputación, entre otros.

Por su papel estratégico en la dinámica social de la organización, los activos intangibles son componentes que potencializan los resultados, es decir, catalizadores de las funciones prioritarias de la organización. Esto representa una red de valor estratégico para cualquier organización. Kaplan y Norton (2004), en su mapa estratégico de la organización, muestran cómo los activos intangibles son el soporte social y psicológico para que los procesos funcionales críticos de la organización se desarrollen orientados a sus propósitos.

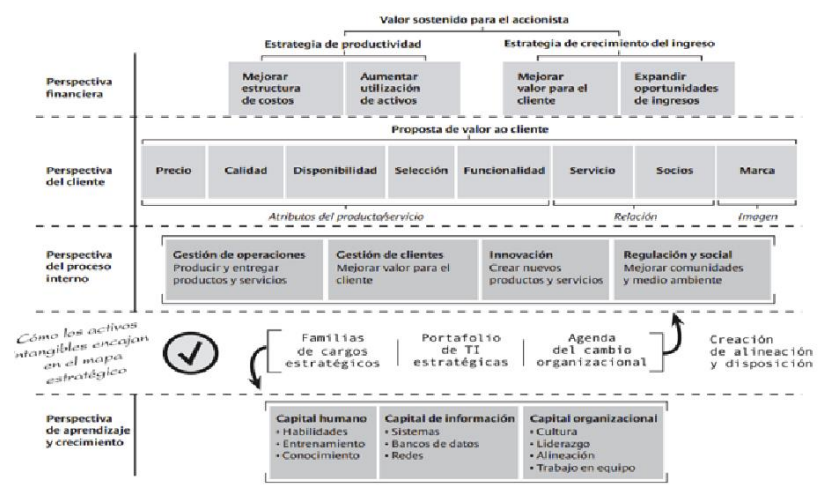

Figura 1. Mapa estratégico organizacional (Kaplan y Norton, 2004).

Los intangibles están ligados a la dimensión macro-sistémica, que son el valor organizacional resultante, por una parte, del esfuerzo operativo de transmitir información; por la otra, del esfuerzo interactivo con sus 
grupos de interés que provoca un nivel de satisfacción y efectividad organizacional; pero fundamentalmente, del esfuerzo sostenido de mejora del sistema, que desencadena en una red de valor compartido. Estas características de los activos intangibles hacen complicada su valoración de forma mecanicista y material. El valor estratégico de los activos intangibles está inserto en la articulación y apoyo a los propósitos de la estrategia corporativa global; además, tiene la capacidad de potencializar los objetivos funcionales $y$, finalmente; agiliza y dinamiza las metas operativas.

El sistema comunicacional debe buscar articular las dimensiones estructurales, funcionales, relacionales $\mathrm{y}$ simbólicas de la organización, de tal forma que genere valor en todos los componentes de la red. La creación de redes de valor se desarrolla a partir de lo que Nosnik (2012) llama el nivel productivo de la comunicación, en el que se da un uso inteligente a la información, con la finalidad de provocar un cambio o innovación en el sistema organizacional que trascienda al supra y macro-sistema.

Por ejemplo, la satisfacción y lealtad del cliente, en corto plazo no incrementa directamente el monto de las ventas de manera significativa, es más, en ocasiones los márgenes de utilidad pueden reducirse. Sin embargo, a largo plazo logra una cartera de clientes consolidada y una tendencia de ventas sostenibles, lo que se traduce en un incremento en la liquidez que abre la oportunidad para invertir en la formación del personal y la calidad en el producto y el servicio, generando mayores ventas, márgenes de utilidad y sustentabilidad financiera. Para ello, es fundamental interpretar la producción simbólica del cliente; escuchando los discursos colectivos; entendiendo sus necesidades; articulando los patrones de comportamiento al producto; acoplando las representaciones sociales a los esfuerzos publicitarios y; respondiendo a las demandas de los múltiples escenarios y factores que interactúan en el contexto, para detonar procesos de articulación a nivel macro-sistémico.

La interpretación de la producción simbólica del sistema social de la organización, va más allá del contenido del mensaje, implica el reconocimiento del significado y el sentido que los agentes organizacionales le confieren a la situación. Así es como se trata de identificar las "relaciones de significatividad" (Bendezu, 2014, p. 260).

\section{Proceso dinamizador del valor de las relaciones sociales: comunicación estratégica}

La Comunicación Estratégica se fundamenta en la perspectiva sistémica de Luhmann (2014), que concibe a la organización como "un sistema social de relación y la comunicación como el centro constructivo de la vida social del sistema" (p. 301). Además, desde la Nueva Teoría Estratégica (NTE) de Pérez (2009), que define a la estrategia como una capacidad humana contribuyente a la articulación de acciones que logran satisfacer las demandas del entorno. Y, finalmente, se nutre del pensamiento comunicacional latinoamericano, desde el que Galindo (2011) propone a la comunicación estratégica como un modelo "para la convivencia y la cooperación ecológica universal” (p. 8).

Partiendo de ahí, intervenir a las organizaciones es una opción que tienen los seres humanos para modificar las estructuras del entorno social. Y la comunicación estratégica, por su naturaleza simbólica y relacional, se concibe como una función del sistema para solucionar problemas de articulación, mediación y negociación; Nosnik (2012) afirma que: "La comunicación es fundamental para que una organización pueda convertirse en una herramienta efectiva de construcción social"(p. 13).

En un sistema la comunicación es una función que dinamiza, orienta y maximiza las relaciones y, en las organizaciones, potencializa la capacidad relacional para generar su desempeño efectivo y sustentable. La comunicación estratégica logra "su máximo nivel productivo en el momento en que los individuos y colectivos se unen entorno al diálogo para innovar" (Nosnik, 2012, p. 20). Para lograrlo se requiere de esfuerzos colectivos sintetizados en procesos de transmisión, distribución y uso inteligente de la información, realizados por un sistema de convivencia que busca generar como retroalimentación beneficios a nivel individual, organizacional y social.

\section{Modelo ICA: la inteligencia comunicacional en la organizaciones}

Para gestionar la comunicación estratégica con la finalidad de construir una red de "inteligencia comunicacional" (Nosnik, 2012 p. 21), es necesario abordar la realidad 
organizacional con metodologías que permitan una comprensión integral y holística de las relaciones sistémicas, para así lograr la implementación de estrategias eficientes y productivas en la construcción de organizaciones comunicacionales.

El modelo ICA, que se presenta a continuación permitirá a las organizaciones restablecer su equilibrio dinámico, adaptando y re-inventando sus patrones de comportamiento, su producción simbólica y sus actividades operativas. Así es posible potenciar su riqueza socio-simbólica y articularla a los propósitos organizacionales, formando una comunidad de inteligencia comunicacional que incida en la creación de una red de valor (Nosnik, 2012). El modelo ICA considera tres fases: investigación, comunicación y acción.

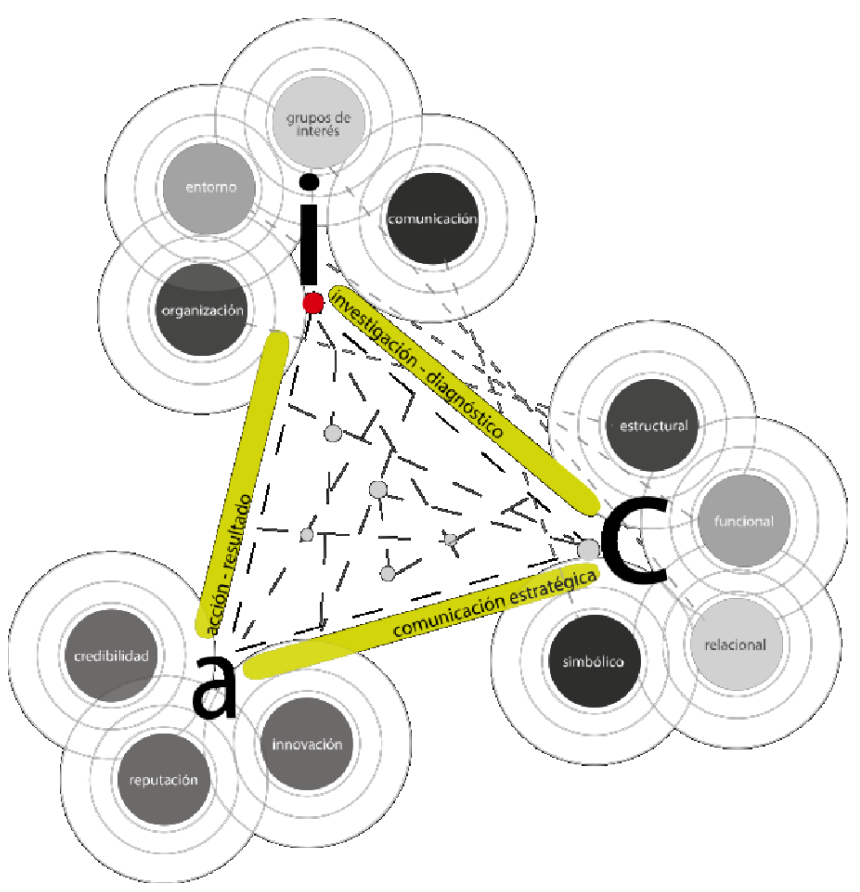

Figura 2. Modelo ICA, elaboración propia.

\subsection{Fase 1: investigación y diagnóstico}

En esta fase se entiende a la investigación como un proceso sistemático y dinámico de diagnóstico del fenómeno organizacional para comprenderlo y resolver problemas prácticos de significación y articulación. Para lograr un acercamiento integral se proponen cuatro modelos de análisis sistémicos: subsistemas organizacionales, factores del entorno, grupos de interés y sistema comunicacional. Para finalmente, realizar un diagnóstico estructural, funcional, relacional y simbólico del sistema que permita diseñar la Estrategia Comunicacional Corporativa (ECCo).

\subsubsection{Análisis estructural: los subsistemas de la organización}

Para analizar la estructura sistémica de la organización, Kast y Rosenzweig (2004. p. 117) proponen estudiar el entramado de relaciones que suceden entre los subsistemas que se encuentran en interacción para la satisfacción de las demandas del entorno.

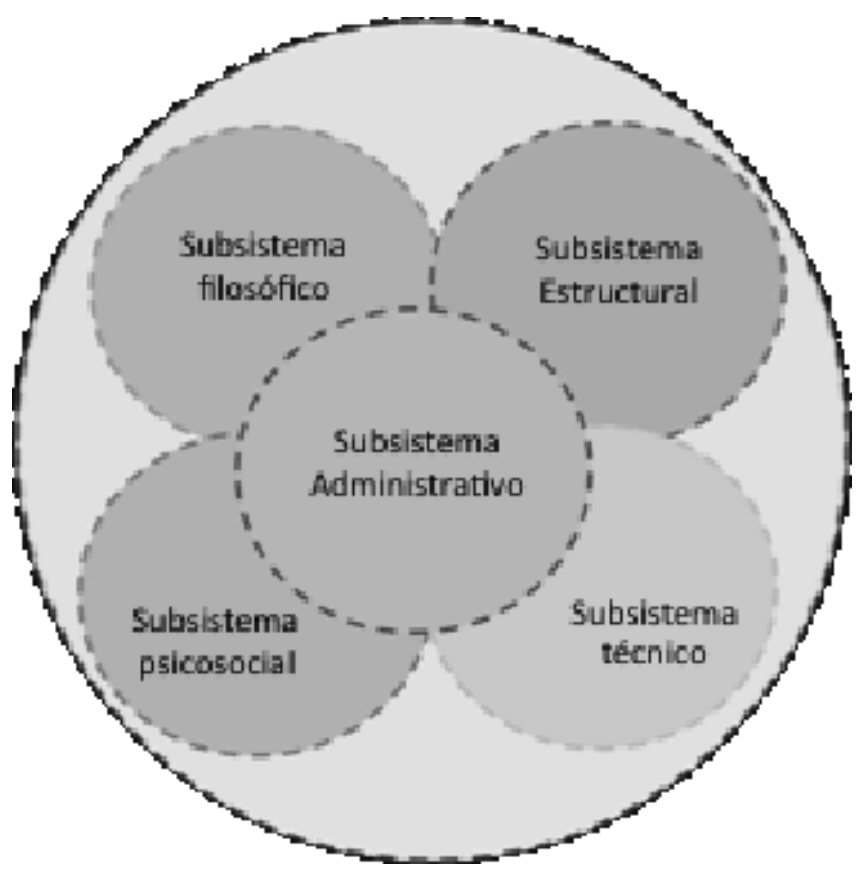

Figura 3. Modelo de análisis de subsistemas (Kast y Rosenzweig, 2004, p. 117).

- Subsistema filosófico: mito fundacional, misión, visión, valores y políticas.

- Subsistema estructural: organigrama, manuales y formatos administrativos.

- Subsistema técnico: productos o servicios que ofrece cada organización.

- Subsistema psicosocial: clima y cultura organizacional, actitudes y expectativas. 
- Subsistema administrativo: objetivos, planes operativos y procesos de control (Kast y Rosenzweig, 2004, pp. 117-121).

El análisis estructural permite apreciar cómo es que se presentan las relaciones intra-sistémicas, su interdependencia entre el sistema organizacional en una lógica de operatividad y eficiencia, atendiendo al entorno donde se circunscribe la organización.

\subsection{Análisis Funcional: factores del Macro-sistema y supra-sistema}

Requiere visualizar a la organización y su entorno, en donde se reconoce a la organización como un sistema que funciona para dar respuesta a las demandas de otros dos sistemas mayores, el supra y el macro-sistema. Para ello, Scheinsohn (2009) propone considerar los siguientes elementos del entorno:

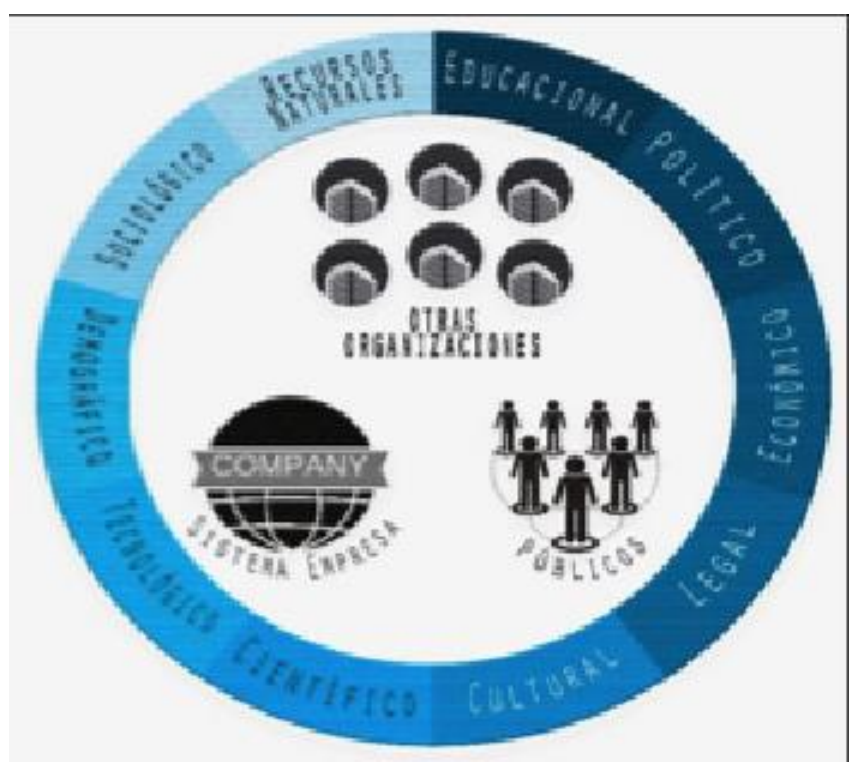

Figura 4. Modelo de análisis del Macrosistema y Suprasistema (Scheinsohn, 2009).

El supra-sistema contiene a los grupos de interés que interactúan con la organización y, por otra parte, estos se relacionan con los componentes del macro-sistema, entre los que se encuentran factores culturales, demográficos, económicos, tecnológicos, científicos, sociales, educacionales, políticos, legales, recursos naturales, entre otros (Scheinsohn, 2009).
Este análisis ayudará a identificar cómo la organización desarrolla funciones para responder a las exigencias externas. Por lo tanto, la efectividad de la organización requiere un sistema de gestión que cumpla con sus objetivos operativos y funcionales y, para ello, debe construir vínculos sociales fuertes de colaboración, participación e integración para responder a las demandas del supra y macro-sistema.

\subsection{Análisis relacional: red de los grupos de interés}

El análisis relacional tiene como finalidad identificar el mapa de vínculos de la organización y sus grupos de interés. Freeman (2004) señala que es necesario lograr una relación integral y estratégica con los stakeholders y/o grupos de interés, para lograrlo se propone la identificación, jerarquización y gestión diferenciada de cada uno de ellos.

1. Identificación: reconocer a los individuos $\mathrm{u}$ organizaciones que tengan una interacción directa o indirecta y agruparlos. Para Capriotti (2009) es necesario identificar los grupos en tres ámbitos: entorno Interno (quienes ejecutan el proceso productivo base), entorno de trabajo (quienes contribuyen con algún insumo al proceso productivo) y entorno general (quienes se relacionan de manera indirecta con el proceso productivo).

2. Jerarquización: debido a la diversidad y amplitud de la red de grupos de interés, es necesario reconocer el impacto que tiene cada uno de ellos con base en diversos criterios: responsabilidad, influencia, cercanía, dependencia, representación, interés, expectativas, control de información, entre otros (Capriotti, 2009, p. 177). Con ello se puede valorar el tipo y densidad de la relación con el objetivo de establecer tres tipos de grupos de interés: claves -vínculo con densidad alta-; secundarios -vínculo con densidad media determinada por la información, el ejercicio del poder y/o actitud-; y terciarios o apáticos -vínculo débil- (Capriotti, 2009, p. 178).

3. Gestión diferenciada: debido a su posición en la matriz de análisis se puede precisar cuáles son los grupos que tienen mayor impacto para la organización y, tomando en cuenta el tamaño, la cercanía, influencia o control de información, es posible determinar los elementos 
necesarios para construir una relación significativa y productiva. De esta manera es posible diseñar la estrategia estratificada de comunicación que integrará la Estrategia Comunicacional Corporativa (ECCo).

\subsection{Análisis simbólico: socio-comunicacional}

Para el análisis socio-comunicacional se requiere la descripción de la estructura, densidad y significado de los vínculos sociales en el sistema organizacional. Herrera \& Bendezú (2017, p 148), conciben a "la comunicación como una interacción simbólica" y que en la organización cobra vital importancia dada su cualidad relacional y social.

Es necesario analizar el sistema socio-comunicacional que estructura un entramado de interacciones simbólicas, para construir estrategias que articulen la producción simbólica del sistema social a los propósitos organizacionales. Al respecto, Manucci (2006), destaca que el propósito de la comunicación estratégica en la organización es la construcción de sentido de los procesos corporativos (p. 58).

Desde esa perspectiva, el análisis socio-comunicacional de Herrera \& Bendezú, (2017) se centra en identificar la densidad de los vínculos sociales y su grado de articulación a los propósitos organizacionales. El análisis considera cuatro categorías:

1. Conexión: identificación de quienes interactúan, su estructura, el tamaño y forma de la red.

2. Relación y vínculo: valoración del vínculo afectivo/emotivo de las relaciones.

3. Articulación de la diferencia: validación de las conexiones lejanas y antagónicas que presentan diferencias funcionales.

4. Sentido y significación: valoración de la relación con los propósitos organizacionales (Herrera \& Bendezú, 2017, p 147).

Con la identificación de la diversidad de interrelaciones, la valoración de la densidad de los vínculos y la descripción de la producción simbólica, es posible gestionar de manera estratégica, los sistemas de comunicación. Describir, interpretar y articular esta dimensión simbólica deberá ser el punto de partida del Modelo ICA.

\section{Diagnóstico}

La fase de investigación permite configurar el mapa sistémico a nivel estructural, funcional, relacional y simbólico. En el modelo de análisis sistémico se pueden identificar la diversidad de relaciones operativas y simbólicas que se configuran a partir de los procesos de significación que comparte, voluntaria e involuntariamente, la organización con sus componentes y su entorno (Scheinsohn, 2009). Ello requiere observar de manera integral y profunda las configuraciones estructurales, funcionales, relacionales $\mathrm{y}$ comunicacionales en el sistema organizacional.

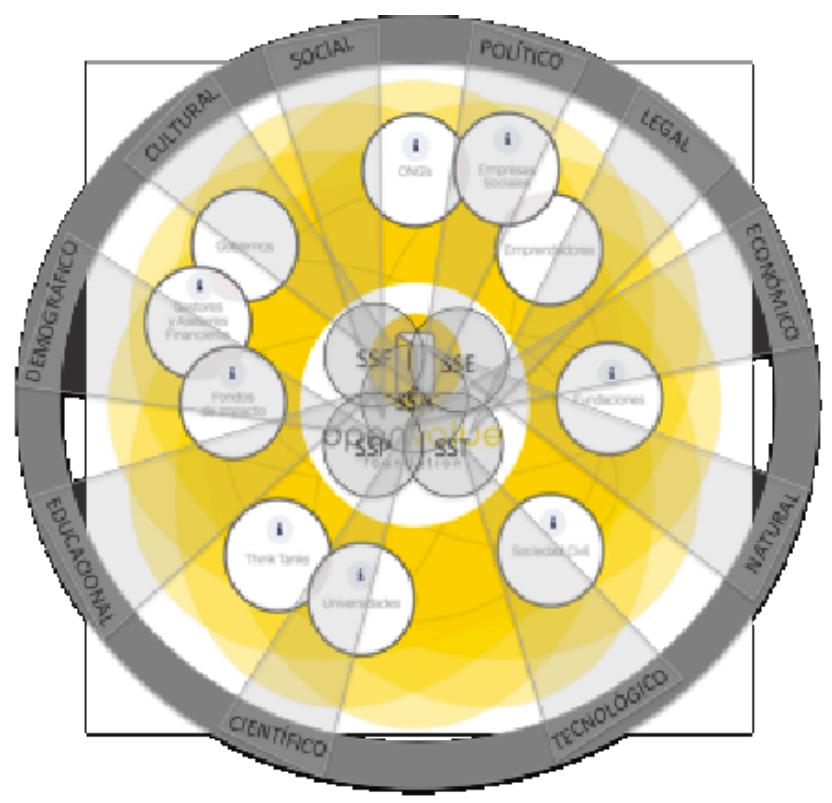

Figura 5. Modelo de análisis sistémico de la organización, elaboración propia (Basado en Kast Kast \& Rosenzweig, 2004; Scheinsohn, 2009 y Capriotti, 2009).

Para la concluir el diagnóstico se elabora una matriz FODA, un modelo situacional útil para la toma de decisiones. La identificación de las fortalezas, amenazas, debilidades y oportunidades en torno a algún problema de articulación, significación y/o sentido detectado en la fase de investigación, permite tomar decisiones estratégicas que den solución a las brechas comunicacionales (Garrido, 2006; Johnson, Scholes, Wittington, 2006).

$\mathrm{Su}$ principal función es identificar las posibles relaciones entre las variables internas y externas. Además, en el marco del modelo ICA, es necesario enfocarse en las brechas 
de relación y articulación que guardan las dimensiones estructurales, funcionales, relacionales y simbólicas del sistema organizacional, para así determinar los objetivos estratégicos que serán el punto de partida del diseño de la Estrategia Comunicacional Corporativa (ECCo).

\subsection{Fase 2: comunicación y estrategia}

Concluida la identificación de las brechas estructurales, funcionales, relacionales y simbólicas que son necesarias para la articulación de los objetivos operacionales, funcionales y comunicacionales de la organización, es momento del diseño de la Estrategia Comunicacional Corporativa (ECCo). La visión de la comunicación estratégica propone observar a la organización como un sistema donde la importancia recae en crear ecologías o "ambientes ricos en información” (Nosnik, 2012, p. 25), que permitan una relación productiva entre todos los componentes del sistema y esto, en una organización, se logra cuando se armoniza la producción simbólica a la operativa, funcional y corporativa.

Scheinsohn (2009), define la comunicación estratégica como la ecología que hace posible la gobernabilidad y el desarrollo de la organización. La ecología es un patrón de configuración social basado en la interacción-colaboración, que plantea la relación con todos los componentes, con el propósito de conformar vínculos colectivos que constituyan un sistema participativo; su finalidad es alinear esos esfuerzos al bienestar individual, organizacional y social para lograr la sustentabilidad del ecosistema. Alcanzar un nivel estratégico significa acoplar las diversas ecologías entre la organización, sus grupos de interés y el entorno.

Es necesaria la planificación de los procesos comunicacionales con una visión multidimensional que considere todos los recursos tangibles e intangibles, la dirección de los procesos de comunicacionales a nivel estructural, funcional, relacional y simbólico, y el impacto en los indicadores de credibilidad, reputación e innovación del sistema. Para ello, se necesita considerar el sistema de comunicación en la organización como un componente que dinamiza y articula la diversidad de dimensiones de la organización entre las que se pueden mencionar:

La Estrategia Comunicacional Corporativa (ECCo) debe desarrollar espacios vinculantes de sus agentes, actividades y sus dimensiones, que le permita a la organización interactuar

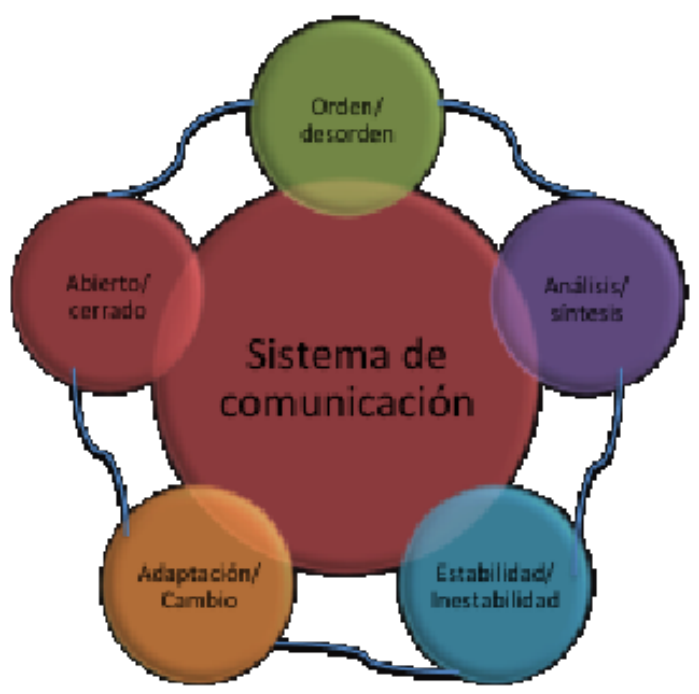

Figura 6. Sistema de Comunicación, elaboración propia basada en (Serrano et al. 2012).

de manera holística con la intención de lograr la convivencia armónica trascendente a nivel individual, organizacional y social. La interpretación de la producción simbólica de los grupos de interés debe acoplarse, a la gestión de los esfuerzos y capacidades comunicacionales, tanto de la organización como de ellos mismos, para que permitan configurar ecosistemas propicios que generen de manera sistemática relaciones productivas.

Estrategia Comunicacional Corporativa (ECCo) es un plan general que gestiona "un conjunto de procesos que son diferentes formas de representar la información" (Nosnik, 2012 p. 56), estos procesos buscan integrar diversas plataformas y dimensiones de comunicación que interactúan entre sí y, al mismo tiempo, buscan la relación entre todos los componentes del sistema para crear una red dinámica. Nosnik (2012) establece los siguientes subsistemas de comunicación en una organización:

Con la finalidad de lograr una ECCo integral y compleja, es necesario provocar espacios de relación que se orienten a los propósitos organizacionales, para ello, se sugiere considerar programas diferenciados que atiendan la diversidad de los grupos de interés, planes que interrelacionen los diversos subsistemas comunicacionales, y además que articule la dimensión estructural, funcional, relacional y simbólica de la organización. 


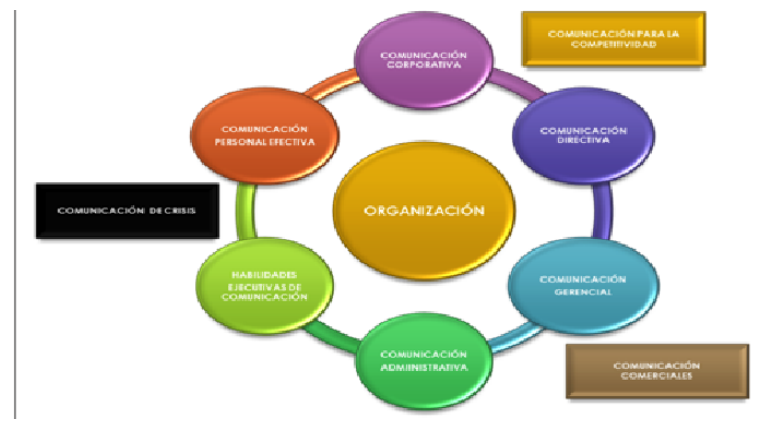

Figura 7. Plan General de Comunicación, elaboración propia basado en (Nosnik, 2012).

\subsection{Fase 3: acción y resultados}

Las implicaciones estratégicas del modelo de gestión de comunicación ICA, se determinan en el grado en que el conjunto de activos tangibles e intangibles contribuyen al desempeño de los procesos internos críticos y, por lo tanto, su valor para la organización. Se trata de demostrar de qué forma la producción simbólica natural del sistema organizacional se convierte en activos intangibles que dinamiza el ECCo, y que influyen en el desempeño de los procesos internos críticos de la organización. Es necesaria la supervisión de la Matriz relacional de indicadores (MaReI) en la que se deben identificar tres tipos de indicadores:

1. Eficacia (operativo). Indican la oportunidad y pertinencia de la comunicación en el logro de las metas operativas.

2. Eficiencia (funcional). Indican el grado de cumplimiento de los objetivos en relación con los recursos financieros, materiales y humanos establecidos.

3. Efectividad (estratégico). Miden el impacto de la ECCo en el entorno y sus grupos de interés. Establecen el grado de trascendencia de los esfuerzos vinculantes en el entorno y los grupos de interés.

La comunicación estratégica, es un espacio de interacción simbólica que tiene como centro la resolución de problemas organizacionales que se generan y solucionan a partir de las interrelaciones de los componentes del sistema organizacional. Las implicaciones de estos principios en el modelo ICA para la gestión de la comunicación estratégica son:
1. Calidad de la información y la retroalimentación;

2. Tácticas diversificadas de vinculación productiva y dialógica;

3. Capacidad de creatividad, innovación y aprendizaje en la solución de los problemas organizacionales y sociales (Nosnik, 2012, p. 75).

Por lo que sus indicadores de efectividad se observan en procesos de construcción colectiva y productiva, como son credibilidad, compromiso, confianza, reputación, creatividad, aprendizaje e innovación. Estos indicadores son activos intangibles que se generan en la interacción de la diversidad de la producción simbólica $\mathrm{y}$, a su vez, inciden en la realidad organizacional a partir de sus relaciones sociales. En este sentido, la ECCo debe considerar tres indicadores comunicacionales estratégicos que Nosnik (2012) describe como tres principios:

1. Credibilidad: resultado del cumplimiento del principio de integridad o ser íntegro, que se refiere a la posibilidad de contrastar el contenido del discurso con el comportamiento organizacional, sus acciones corporativas y sus resultados.

2. Reputación: se refiere al hecho de satisfacer las necesidades y expectativas de los grupos de interés que genera una buena imagen y reputación, esto es el resultado del cumplimiento del principio de ser integral.

3. Innovación: se traduce la generación de cambios y mejoras en beneficio de la organización, los grupos de interés y su entorno social. Ello se logra en el cumplimiento del principio de estar integrado a la productividad del sistema a nivel micro, supra y su macro-sistema (Nosnik, 2012, p. 35-36).

\section{Conclusiones}

El Modelo de gestión comunicacional ICA busca armonizar los contrastes de la producción estructural, funcional, relacional y simbólica de la organización. La armonización resulta de una construcción de interacciones coordinadas, tanto por integraciones y diferenciaciones de la organización. Para Massoni (2004), gestionar la comunicación 
Tabla 1. Matriz relacional de indicadores (MaReI), elaboración propia basado en (Kaplan y Norton, 2004).

\begin{tabular}{|c|c|c|c|c|}
\hline Clour & Eficacia & Eficiencia & Efectividad & Dimensión \\
\hline & Logro de objetivos & Logro de costos & $\begin{array}{l}\text { Impacto en públicos y } \\
\text { entorno }\end{array}$ & Comunicaciona \\
\hline Financiera & Aumento de ingresos & $\begin{array}{lll}\text { Retorno } & \text { de } & \text { la } \\
\text { inversión } & & \\
\end{array}$ & $\begin{array}{l}\text { Satisfacción de los } \\
\text { accionistas }\end{array}$ & $\begin{array}{l}\text { Credibilidad y } \\
\text { reputación }\end{array}$ \\
\hline Relación-cliente & Cuota del mercado & Incremento de ventas & $\begin{array}{lll}\begin{array}{l}\text { Lealtad } \\
\text { clientes }\end{array} & \text { de } & \text { los } \\
\end{array}$ & $\begin{array}{l}\text { Confianza y } \\
\text { competitividad }\end{array}$ \\
\hline Procesos críticos & $\begin{array}{lll}\text { Aumento en la } \\
\text { producción }\end{array}$ & $\begin{array}{l}\text { Decremento en el } \\
\text { desperdicio }\end{array}$ & Satisfacción laboral & $\begin{array}{l}\text { Colaboración } \\
\text { y } \\
\text { productividad }\end{array}$ \\
\hline Aprendizaje-Crecimiento & $\begin{array}{l}\text { Incremento en la } \\
\text { capacitación }\end{array}$ & $\begin{array}{l}\text { Diversificación en los } \\
\text { productos }\end{array}$ & $\begin{array}{l}\text { Innovación en el } \\
\text { mercado }\end{array}$ & $\begin{array}{l}\text { Aprendizaje y } \\
\text { creatividad }\end{array}$ \\
\hline
\end{tabular}

de manera estratégica es un proceso muy complejo y con alto potencial, que logra que cualquier situación "se vuelva más gobernable" (p. 20).

El Modelo ICA se centra en la vinculación comunicacional, es decir, la interacción dialógica, la acción colectiva y la participación social. En la fase de investigación y diagnóstico, es necesario llegar a descubrir la diversidad simbólica organizacional y la relación con su dimensión estructural, funcional y relacional. En la fase de comunicación y estrategia, la ECCo deberá surgir de las interacciones simbólicas, para lograr la implicación de los integrantes del sistema y, que ese compromiso, sea una fuerza vital que potencialice las redes de valor; además, los esfuerzos comunicacionales deben lograr articular los recursos simbólicos, relacionales y culturales a los componentes materiales, financieros, tecnológicos, para hacerlos complementarios. Finalmente, en la fase de acción y resultados, los indicadores de eficiencia del modelo de gestión ICA se centran en la generación de activos intangibles, como la credibilidad, reputación e innovación.

El Modelo ICA, asume a la comunicación como una función del sistema que desarrolla la posibilidad del diálogo inteligente, la habilidad de articulación de la diversidad y la capacidad de orientar la producción simbólica en beneficio de los individuos, las organizaciones y la sociedad. La aportación del modelo ICA en la gestión de la comunicación estratégica se traduce en la oportunidad de lograr la construcción participativa, productiva y sustentable de la organización. Esto se logrará usando metodologías que permitan una comprensión integral y holística de las relaciones sistémicas, para la implementación de estrategias eficientes y productivas en la construcción de organizaciones comunicacionales que respondan a las demandas del entorno actual.

\section{Referencias}

Bendezú, R. (2014). Claves para identificar un problema de comunicación estratégica. En R. Herrera y R. A. Pérez (coordinadores), Nueva Teoría Estratégica: El paradigma emergente para la co-construcción y transformación de la realidad (pp. 241-267). Santiago de Chile: Santillana.

Bourdieu, P. (2002). Estrategias de reproducción y modos de dominación. Colección Pedagógica Universitaria, 37-38.

Bourdieu, P. \& Wacquant, J. D. (1995). Respuestas: Por una antropología reflexiva. México: Grijalbo.

Capriotti P. P. (2009). Branding Corporativo: fundamentos para la gestión estratégica de la Identidad Corporativa. Librería de la Empresa, Chile.

Freeman, R. E. (2004). Strategic Management: A stakeholder approach. Boston.

Galindo, L.J. (2011). Comunicación Estratégica e ingeniería social en comunicación. Revista Razón y Palabra. $n^{o} 75$. México

Garrido, S. (2006). Dirección estratégica. $7^{\mathrm{a}}$. edición. Madrid: McGraw Hill.

Herrera, E., R. \& Bendezú, U., R. (2017). La estrategia desde una matriz comunicacional. En Arribas, A., Herrera, E., R. \& Pérez, R., A. (Coordinadores), Nueva Teoría Estratégica: Repensando la estrategia desde la comunicación, (pp. 135-167). Razón y palabra sello editorial.

Johnson, G., Scholes K., \& Whittington, R. (2006). Dirección estratégica. $7^{\mathrm{a}}$. edición. Madrid: Pearson Educación. 
Kaplan, R.S. y Norton D.P. (2004) Medir la disposición estratégica de los activos intangibles. Harvard Bussiness Review America latina.

Kast, F., \& Rosenzweig, J. E. (2004). Administración en las organizaciones. Editorial McGrawHill.

Luhmann, N. (2014). Introducción a la teoría de sistemas. México. Universidad Iberoamericana/ Alianza editorial.

Manucci, M. (2006): La estrategia de los cuatro círculos. Bogotá: Norma.

Massoni (2004). El aporte de la comunicación estratégica en las organizaciones. En: Herrera R. \& Pérez, R. A. (2014). Nueva Teoría Estratégica: El paradigma emergente para la co-construcción y la transformación de la realidad. FISEC. Santiago, Chile.

Nosnik, A. (2012). Teoría de la Comunicación Productiva. Exploraciones más allá de la retroalimentación. Argentina. Homosapiens ediciones.

Nosnik, A. (2012) Análisis de sistemas de comunicación en las organizaciones: 10 años después. En Fernández, C. (2012) La comunicación en las organizaciones. Trillas. México.

Parsons. (2012). The Social System. New Orleans: Q. P. Books, Ed.

Pérez R.A, \& Massoni, S. (2009): Hacia una teoría general de la estrategia. Barcelona: Ariel.

Pérez, R. A. (2012). Pensar la estrategia. Buenos Aires: La Crujía.

Rodríguez, D., \& OPazo, M. (2007). Comunicaciones de la organización. Santiago: Ediciones Universidad Católica de Chile.

Scheinsohn, D. (2009). Comunicación estratégica. Buenos Aires: Ed. Granica.

Serrano, R; Pérez, J; Mendoza, F; Ávila, X; Revilla, M. (2012) La organización habitable. Transición de TEXLMEX, de lo público a lo privado. México. Editorial Fecha. 